\title{
Entornos y actividad física en enfermedades crónicas: Más allá de factores asociados
}

\author{
Environments and physical activity in chronic diseases: Beyond associated factors \\ Diana Isabel Muñoz-Rodríguez ${ }^{*}$ orcid.org/0000-0003-4255-4813 \\ Catalina María Arango-Alzate' ${ }^{1}$ orcid.org/0000-0001-5134-9294 \\ Ángela María Segura-Cardona' orcid.org/0000-0002-0010-1413
}

1 Escuela de Graduados, Universidad CES. Medellín, Colombia.

Fecha de recepción: Marzo 3 - 2017

Fecha de revisión: Febrero 20 - 2018

Fecha de aceptación: Abril $13-2018$

Muñoz-Rodríguez DI, Arango-Alzate CM, Segura-Cardona AM. Entornos y actividad física en enfermedades crónicos: más allá de factores asociados. Univ. Salud. 2018;20(2):183-199. DOI: http://dx.doi.org/10.22267/rus.182002.122

\section{Resumen}

Introducción: Las enfermedades crónicas no transmisibles son la mayor causa de morbimortalidad. La estrategia más costo-efectiva es la realización de actividad física pero hay una relación directa entre el incremento de estas enfermedades con la alta prevalencia de personas físicamente inactivas. Objetivo: Recoger los elementos esenciales de las enfermedades crónicas en términos del impacto y sus efectos negativos y mostrar, dentro de los factores de riesgo, a la inactividad física como uno de los determinantes clave en su aparición y el rol de los entornos construidos en esta conducta no saludable. Materiales y métodos: Estudio descriptivo mediante revisión documental sobre el papel de los entornos como factores determinantes entre la inactividad física y el desarrollo de las enfermedades crónicas no transmisibles. Resultados: Se describen los efectos negativos de las enfermedades crónicas, los factores que influyen en su aparición y se destaca a la inactividad física como uno de los determinantes de las enfermedades crónicas. La evidencia empírica sugiere que las características del entorno obran como determinantes en este comportamiento dentro de los estilos de vida. Conclusión: Los entornos en que habitan las personas se constituyen en factores asociados y determinantes en la realización o no de actividad física y deben ser considerados, más allá de los factores individuales, si se quiere tener éxito en la prevención y control de las enfermedades no transmisibles.

Palabras clave: Actividad física; enfermedades crónicas; entorno construido; factores epidemiológicos. (Fuente: DeCS, Bireme).

\begin{abstract}
Introduction: Chronic noncommunicable diseases are the major cause of morbidity and mortality. The most costeffective strategy is the practice of physical activity but there is a direct relationship between the increase of these diseases with the high prevalence of physically inactive people. Objective: To collect the essential elements of chronic diseases in terms of impact and their negative effects and to show, within risk factors, physical inactivity as one of the key determinants in their emergence and the role of environments built on this unhealthy behavior. Materials and methods: A descriptive study was conducted through documentary review on the role of environments as determinants between physical inactivity and the development of chronic noncommunicable diseases. Results: The negative effects of chronic diseases are described as well as the factors that influence their appearance. Physical inactivity is highlighted as one of the determinants of chronic diseases. The empirical evidence suggests that the characteristics of the environment work as determinants in this behavior within the lifestyles. Conclusion: The environments where people inhabit are associated factors and determinants in the practice or not of physical activity and should be considered, beyond the individual factors, if there is the need to succeed in the prevention and control of noncommunicable diseases.
\end{abstract}


Keywords: Exercise; chronic disease; built environment; epidemiologic factors. (Source: DeCS, Bireme).

\section{Introducción}

Las enfermedades crónicas no transmisibles (ECNT) son lesiones de larga duración, característica que las hace complejas por su permanencia en el tiempo y que además, generan discapacidad residual en quienes la padecen y en sus familias, llevando al deterioro de sus funciones físicas y sociales; por tanto, el impacto negativo de una ECNT se traslada a la familia y a la comunidad que deben cuidar del enfermo, asistir y suplir las funciones que éste deja de realizar(1,2), generando una cadena de dependencia funcional desde la asistencia en las funciones básicas cotidianas, hasta las económicas y sociales.

Además de las secuelas que generan estas enfermedades en los individuos, sus familias y la comunidad, su impacto sobre el sistema por los costos que demanda, el deterioro de la calidad de vida y la pérdida de años de vida saludables, las constituyen en un grave problema para la salud pública pues se consideran las mayores aportantes a la morbimortalidad y discapacidad mundial $(3,4)$.

Este grupo de enfermedades ha sido ampliamente estudiado desde hace muchos años, pues con los cambios epidemiológicos que se dieron posterior al control de las enfermedades infecciosas, éstas empezaron a explicar la carga mundial de enfermedad y la fuerte asociación con cuatro factores de riesgo modificables a saber: consumo de tabaco, de alcohol, dietas malsanas y la inactividad física(5). Esta última, llama la atención pues su control, denominado realización de actividad física, ha demostrado ser una de las intervenciones más costo-efectivas, de muy bajo costo, en muchas ocasiones gratuita, equitativa, y con beneficios para la salud general(6). La actividad física (AF) es, "cualquier movimiento voluntario que contribuye al gasto energético total del ser humano", incluye el movimiento de todos los músculos grandes para cualquier propósito, realizados a lo largo del día(7). Según el Colegio Americano de Medicina Deportiva (ACSM por sus siglas en inglés), una vez el individuo se mueve voluntariamente, aumenta su metabolismo como producto de la actividad muscular(8,9).

A nivel mundial, se han estructurado redes y programas para el fomento de conductas saludables como la realización de la AF; así, el "Conjunto de Acciones para la Reducción Multifactorial de las Enfermedades Crónicas no Transmisibles, CARMEN"(10), es una de las estrategias más reconocidas y recomendadas por la acción conjunta de la Organización Mundial de la Salud (OMS), la Organización Panamericana de Salud (OPS), los Centros para el control y prevención de enfermedades (CDC por sus siglas en inglés), y por los gobiernos que hacen parte de los acuerdos para el control de las ECNT dentro de los que está Colombia. También desde los CDC, se han construido políticas para ayudar a reducir la carga de morbilidad crónica a través de estrategias con enfoque comunitario(11).

Algunos programas han sido pioneros y los países se han acogido a las recomendaciones que OMS y centros de apoyo han brindado(12-14). Sin embargo, y a pesar de la amplia evidencia en diversas formas de control de estas enfermedades tanto para su prevención como para el tratamiento, los resultados esperados de su reducción no ha sido el esperado. Si las condiciones permanecen como están hasta ahora, la tendencia es hacia el incremento de la exposición a los factores de riesgo y de los desenlaces relacionados con este grupo de enfermedades(15).

La evidencia de estas condiciones clínicas, reconocidas como de alto costo, ha mostrado que, si se controlan los cuatro factores de riesgo descritos, la morbimortalidad y el impacto negativo que estas producen, podría reducirse. Los mayores aportantes a este tipo de enfermedades han sido los países de bajos y medianos ingresos, donde las condiciones de pobreza, entre otros componentes de los entornos, pueden obstaculizar el consumo de dietas saludables y donde se sabe de la fuerte asociación que hay entre estratos 
socioeconómicos bajos, constituidos por la mayoría de la población, con alto consumo de alcohol y cigarrillo(4,16). Las intervenciones para reducir la exposición a estos factores de riesgo, han implicado retos importantes y una alta inversión económica, pues dependen, entre otros, de cambios culturales y multisectoriales que mejoren las condiciones socioeconómicas de la población y su poder adquisitivo para garantizar mejores condiciones de alimentación y para reducir el consumo de alcohol y cigarrillo.

Por tanto, considerando que la realización de $\mathrm{AF}$ está presente en la cotidianidad de las personas dentro de sus actividades domésticas, de tiempo libre, recreativas y como formas de trabajo(17), se esperaría que todos o al menos el $80 \%$ de la población mundial hiciera la $\mathrm{AF}$ recomendada por la OMS (por lo menos 150 minutos de actividad física semanal a intensidad moderada o vigorosa), pues adherirse a esta estrategia ayudaría a prevenir casos de muertes por ECNT, la mayoría prematuras, así como a reducir la frecuencia de su aparición $(6,18)$, sin que genere costos elevados para las personas, sus familias ni el sistema; una revisión sistemática en 2014 concluyó que las intervenciones para mejorar las oportunidades para caminar y andar en bicicleta parecen aumentar la actividad física de manera rentable ${ }^{(19)}$. No obstante, la prevalencia de AF es muy baja en todo el mundo (menos del $40 \%)^{(20)}$ y las razones que explican este comportamiento poco saludable se han centrado hasta ahora, en una evidencia contundente sobre los factores individuales que, si bien han contribuido a la explicación de una parte de este fenómeno denominado inactividad física, no han logrado modificar sus prevalencias 21,22$)$.

Esto, ha llevado al cuestionamiento permanente sobre la inactividad física y alrededor del mundo se han creado paradigmas y posibles explicaciones dentro de las que, en las últimas décadas ha surgido con fuerza, bajo los modelos conceptuales ecológicos y de la epidemiología social(23-25), la inclusión de características que son proximales a las personas y distales a ellas, tales como los entornos, que contribuyan a la explicación y comprensión de la inactividad física; entender por qué muchas personas son inactivas, considerando que una proporción importante (más del 80\%) reconoce los beneficios de esta práctica para la salud general(17,26,27), es un reto de los países a nivel mundial $y$ especialmente de aquellos en desarrollo, para lograr la toma de decisiones más razonables en las adecuaciones de estrategias que incrementen la prevalencia de realización de actividad física, a bajo costo y de manera eficaz(17).

Esta revisión pretende recoger los elementos esenciales de las enfermedades crónicas en términos del impacto y sus efectos negativos y mostrar, dentro de los factores de riesgo, a la inactividad física como uno de los determinantes clave en su aparición. A partir de esto, se documenta sobre el papel de factores distales al individuo que se asocian a las características individuales con su condición de ser físicamente activos o no, tales como los que se derivan de los entornos en que ellos habitan, con el fin de explorar otras posibles causas de la inactividad física, que conduzcan a la toma de decisiones en intervenciones más razonables para países como Colombia.

\section{Materiales y métodos}

Se condujo un estudio de revisión de tema, a través de búsqueda libre durante el año 2016, que incluyó estudios reportados en la literatura relacionados con las enfermedades crónicas, su epidemiología, efectos negativos; sobre la actividad e inactividad física, y su relación con los entornos construidos y percibidos en que habitan las personas. Las bases de datos consultadas fueron aquellas relacionadas con las ciencias de la salud disponibles en los recursos electrónicos bajo la licencia de la Universidad CES. Entre ellas, Pubmed, Ovid, LILACS, Google académico y SciELO; además, fueron incluidos documentos de las páginas oficiales mundiales y nacionales de expertos en la temática tales como OMS, el Centro para el Control y Prevención de Enfermedades, el Observatorio Nacional de Salud y el Ministerio de protección social en Colombia, así como documentos indexados con elementos conceptuales clave. Se incluyeron artículos en idioma español, inglés y portugués, 
privilegiando aquellos con publicación menor a 10 años. Las palabras de búsqueda en castellano fueron principalmente actividad física, enfermedades crónicas, entornos construidos, factores epidemiológicos. Las palabras de búsqueda en ingles fueron: physical activity, Chronic Disease, built environment, epidemiologic factors.

\section{Problemática que engloban las enfermedades crónicas}

Las ECNT se definen como afecciones generalmente de desarrollo lento, de larga duración y causadas por alteraciones patológicas no reversibles, que se controlan pero no se curan $\mathrm{y}$ que por tanto generan discapacidad residual y deterioro de la calidad de vida relacionada con la salud en quienes la padecen(28-30). Estas enfermedades agrupan condiciones clínicas que deterioran sistemas vitales tales como el cerebro, el corazón, el sistema circulatorio entre otros. Los cuatro tipos principales de ECNT son las enfermedades cardiovasculares, el cáncer, las enfermedades respiratorias crónicas, la diabetes, $\mathrm{y}$ en los últimos tiempos se han incluido las enfermedades mentales(29).

Las ECNT constituyen la principal causa de morbimortalidad en adultos en todas las regiones del mundo. De las 58 millones de muertes que se presentaron en el mundo en el año 2005, 35 millones fueron atribuibles a enfermedades crónicas como cardiopatías, cánceres, enfermedades pulmonares, osteomusculares y genitourinarias; prácticamente el doble de las enfermedades infecciosas(31). Para el año 2008, 36 millones de personas murieron de una enfermedad crónica, la mitad fueron de sexo femenino y el $29 \%$ era de menos de 60 años de edad(32). Solamente un 20\% de las muertes causadas por enfermedades crónicas se presentaron en países desarrollados, mientras que el $80 \%$ se registraron en países con niveles de ingreso medio y bajo, en el cual vive la mayoría de la población mundial(33).

Una década después, la evidencia muestra que las condiciones no han cambiado; un informe de la OMS publicado en enero de 2015 reportó que las ECNT producen 38 millones de muertes anuales en el mundo. Casi el $75 \%$ de las mismas provienen de países de bajos y medianos ingresos; 16 millones son prematuras (antes de los 70 años) y de estas, el $82 \%$ son de países en desarrollo. La pobreza está estrechamente relacionada con las enfermedades no transmisibles(31).

Se ha documentado que la carga de las enfermedades crónicas en los Estados Unidos (EU) es atribuible a por lo menos tres factores clave que incluyen: el estilo de vida y otros comportamientos; los factores sociales y ambientales que promueven o afectan negativamente a la salud; y un aumento de la esperanza de vida, que lleva a un incremento en el número de personas mayores con una o más condiciones crónicas y discapacidades afines(11). Según el CDC, las enfermedades, condiciones crónicas y las conductas de riesgo para la salud que las causan, representan la mayoría de los costos de atención de salud; el $86 \%$ de todo el gasto en atención en 2010 fue para las personas con una o más condiciones médicas crónicas(34). Los costos totales de las enfermedades del corazón y derrame cerebral en el mismo año se estimaron en \$315,4 mil millones de dólares, de los cuales $\$ 193,4$ mil millones fueron para los costos médicos directos, sin incluir los costos de la atención clínica de reposo; la atención del cáncer costó \$157 mil millones en dólares de 2010. El costo total estimado de la diabetes mellitus (DM) diagnosticada en 2012 fue de $\$ 245$ mil millones, incluyendo $\$ 176$ mil millones en costos médicos directos y $\$ 69$ mil millones en disminución de la productividad incluyendo los costos por incapacidad asociados a las personas que estuvieron ausentes de sus lugares de trabajo debido a ausencia total o disminución en su capacidad laboral(35).

En julio del año 2015, el Observatorio Nacional de Salud de Colombia publicó la quita edición del informe de Carga de enfermedad por Enfermedades Crónicas no Transmisibles y Discapacidad en Colombia, a partir del cual se estableció la estimación de las mismas, ésta se basó en la combinación de varias fuentes de información a través de la implementación de una serie de modelos estadísticos predictivos. Se 
realizó una revisión de literatura en Colombia y como no se encontró suficiente información, se revisaron estudios en América Latina, que reportaran mediciones en la población sobre parámetros de ocurrencia y mortalidad para un subgrupo de ECNT (enfermedades cardiovasculares, cáncer, diabetes, hipertensión arterial y enfermedad pulmonar obstructiva crónica)(2). La Tabla 1, describe los datos de frecuencia de morbimortalidad de las principales ECNT según el sexo, y en ella se puede observar que, la tendencia de estas enfermedades es hacia su incremento.

Tabla 1. Frecuencia de morbimortalidad derivada de las enfermedades crónicas no transmisibles en Colombia entre los años 2000 y 2014

\begin{tabular}{|c|c|c|c|c|}
\hline ECNT & $\begin{array}{l}\text { Medida de } \\
\text { frecuencia }\end{array}$ & Mujeres & Hombres & Observaciones \\
\hline \multirow[t]{2}{*}{$\begin{array}{l}\text { Hipertensión arterial } \\
\text { (HTA) }\end{array}$} & Prevalencia & $\begin{array}{l}\text { año 2000: } 25,9 \% \\
\text { año 2014: } 31,3 \%\end{array}$ & $\begin{array}{l}\text { año 2000: } 23,8 \% \\
\text { año 2014: } 28,7 \%\end{array}$ & $\begin{array}{l}\text { Departamento con mayor } \\
\text { prevalencia: Antioquia }\end{array}$ \\
\hline & $\begin{array}{l}\text { Tasa de mortalidad } \\
\text { al final de periodo } \\
(2014)\end{array}$ & $\begin{array}{l}12,7 \times 100.000 \\
\text { mujeres }\end{array}$ & $\begin{array}{l}13,7 \times 1000.000 \\
\text { hombres }\end{array}$ & SD \\
\hline \multirow{2}{*}{$\begin{array}{l}\text { Enfermedad } \\
\text { cerebrovascular (ECV) } \\
\text { hemorrágico }\end{array}$} & $\begin{array}{l}\text { Incidencia de ECV al } \\
\text { final de periodo }\end{array}$ & SD & SD & $\begin{array}{l}7,58 \times 100.000 \mathrm{hb} \\
\text { (población general) }\end{array}$ \\
\hline & $\begin{array}{l}\text { Tasa de mortalidad } \\
\text { al final de periodo }\end{array}$ & SD & SD & $\begin{array}{l}18,8 \times 100.000 \mathrm{hb} \\
\text { (población general) }\end{array}$ \\
\hline $\begin{array}{l}\text { Enfermedad } \\
\text { cerebrovascular (ECV) } \\
\text { isquémico }\end{array}$ & $\begin{array}{l}\text { Incidencia al final de } \\
\text { periodo }\end{array}$ & $\begin{array}{l}7,75 \times 100.000 \\
\text { mujeres }\end{array}$ & $\begin{array}{l}17,08 \times 100.000 \\
\text { hombres }\end{array}$ & $\begin{array}{l}\text { Mayor Incidencia en los } \\
\text { mayores de } 50 \text { años }\end{array}$ \\
\hline Cáncer (CA) & $\begin{array}{l}\text { Casos nuevos al final } \\
\text { de periodo }\end{array}$ & $\begin{array}{l}132.799 \\
\text { Más frecuente: seno y } \\
\text { cuello uterino }\end{array}$ & $\begin{array}{l}174.638 \\
\text { Más frecuente: } \\
\text { próstata y colon }\end{array}$ & $\begin{array}{l}\text { Incremento leve de CA de } \\
\text { pulmón en mujeres entre } \\
\text { los años } 2000 \text { y } 2014\end{array}$ \\
\hline \multirow[t]{3}{*}{ Diabetes Mellitis } & $\begin{array}{l}\text { Prevalencia al final } \\
\text { de periodo }\end{array}$ & $4,6 \%$ & SD & SD \\
\hline & $\begin{array}{l}\text { Distribución } \\
\text { porcentual de } \\
\text { muerte }\end{array}$ & $58 \%$ & $42 \%$ & $\begin{array}{l}5650 \text { promedio anual de } \\
\text { muerte población general }\end{array}$ \\
\hline & Tasa de mortalidad & $\begin{array}{l}15,3 \times 100.000 \\
\text { mujeres }\end{array}$ & $\begin{array}{l}13,5 \times 100.000 \\
\text { hombres }\end{array}$ & $\begin{array}{l}\text { Razón de mortalidad Mujer } \\
\text { : Hombre } 1,13\end{array}$ \\
\hline \multirow[t]{2}{*}{ EPOC } & Prevalencia & $\begin{array}{l}\text { 3,3\% por revisión } \\
\text { sistemática } \\
0,7 \% \text { por RIPS }\end{array}$ & $\begin{array}{l}4,1 \% \text { por revisión } \\
\text { sistemática } \\
0,7 \% \text { por RIPS }\end{array}$ & $\begin{array}{l}\text { Prevalencia estable en } \\
\text { ambos sexos a final de } \\
\text { periodo }\end{array}$ \\
\hline & Tasa de mortalidad & $25,8 \times 100.000 \mathrm{hb}$ & $36,6 \times 100.000 \mathrm{hb}$ & $\begin{array}{l}\text { Razón de mortalidad } \\
\text { Hombre:Mujer } 1,4 \\
\text { Mayor riesgo en mayores } \\
\text { de } 40 \text { años }\end{array}$ \\
\hline
\end{tabular}

Fuente: Informe de Carga de enfermedad por Enfermedades Crónicas no Transmisibles y Discapacidad en Colombia. Observatorio Nacional de Salud de Colombia ${ }^{2}$

hb: habitantes.

RIPS: Registro individual de prestación de servicios de salud (Resolución 3374 de 2000)

SD: Sin dato

\section{Efectos negativos de las enfermedades crónicas}

La evidencia ha venido documentando a través del tiempo y de manera consensuada la existencia de una serie de factores que en conjunto explican el inicio, desarrollo y complicaciones de este grupo de enfermedades(14,33). Éstos, se clasifican como no modificables, metabólicos o fisiológicos, y conductuales modificables; en el primer grupo se encuentran factores como la edad, el sexo, la raza, los antecedentes familiares; en el segundo grupo referido a los factores metabólicos, se encuentran hipertensión arterial, sobrepeso, hiperglucemia e hiperlipidemia; y en el último grupo de factores se encuentran aquellos 
modificables(5), dentro de estos, los relacionados con los comportamentales, estilos de vida y hábitos, nivel de estrés e incluso la calidad de vida, explican la alta prevalencia de estas enfermedades. Este grupo de factores está representado por el consumo de alcohol y tabaco, la malnutrición, el sedentarismo y la inactividad física. Según la OMS, alrededor de 3,2 millones de muertes al año, se pueden atribuir a la actividad física insuficiente( ${ }^{(36)}$.

De este grupo de factores de riesgo modificables, también hacen parte aquellos asociados al ambiente y los entornos en que habitan las personas. Se sabe que, las razones de enfermar pueden provenir del propio organismo, pero también de los entornos en que habitan. Así, la urbanización rápida y no planificada, el envejecimiento acelerado de la población, la mundialización de unos modos de vida poco saludables, la globalización en la comercialización, son algunas características de los entornos tales como los barrios, las escuelas y colegios, los lugares de trabajo entre otros, que podrían ayudar a explicar la presencia de estas ECNT, pues son riesgos sobre los que el individuo tiene poco control y sobre los que la influencia de los sectores de la salud es casi nula por los modelos de atención individuales y bajo paradigmas biomédicos(31). Esto es, las características distales a los individuos, propuestos por los modelos sociecológicos(37).

Aunque investigadores y organizaciones mundiales a cargo de la salud de las poblaciones han hecho innumerables esfuerzos por controlar la aparición de estas enfermedades a través de la intervención de factores de riesgo, su presencia y rápido incremento, especialmente en poblaciones de bajos y medianos ingresos, impide el desarrollo de iniciativas que buscan precisamente romper con las cadenas de pobreza, pues en todo el mundo las personas pobres tienen mayor probabilidad de desarrollar enfermedades crónicas y de morir por esa causa. Además, las enfermedades crónicas conllevan un considerable gasto financiero y pueden hundir a individuos y hogares en la pobreza(38,39). Se sabe que "las personas vulnerables y en desventaja social se enferman más y mueren antes que las personas de mayores posiciones sociales, sobre todo porque están en mayor riesgo de estar expuestos a productos y a conductas nocivas, como el tabaco o alimentos poco saludables, a actividades que favorecen el sedentarismo, y que tienen acceso limitado a los servicios de salud", y en quienes "los costos que deben asumir por estas condiciones, agotan rápidamente los recursos del hogar" (31).

Los efectos negativos que producen estas enfermedades trascienden lo biológico y deterioran a las personas, sus familias, el sistema de salud, los países(11). Traen consigo comorbilidades, discapacidad, altos costos de atención, años de vida potencialmente perdidos e incluso el detrimento en la calidad de vida y en el desempeño social de quienes padecen estas condiciones. Se ha documentado, que el impacto de las ECNT produce estrés a nivel financiero, sensación negativa hacia sí mismo y sus capacidades, cambios en la filosofía de vida y problemas de conducta y emocionales, con impacto en los procesos de comunicación e interacciones familiares y con los cuidadores, que se agudizan por los niveles de incertidumbre derivados de las posibles consecuencias de la enfermedad(30).

Con relación a los costos generados por la atención de estas enfermedades (aunque su estimación varía de un país a otro y su medición no ha podido ser estandarizada), se ha documentado que las ECNT representan una carga financiera significativa en los presupuestos de salud y el bienestar de las naciones, que es probable que aumente con el tiempo; los gastos por enfermedad cardiovascular (una de las más frecuentes) ocupan alrededor del $12 \%$ al 16,5\% del gasto total en salud, otras ECNT oscilaron entre 0,7 y $7,4 \%{ }^{(35)}$.

Una revisión sistemática publicada en el año 2015 , se propuso evaluar el impacto de este grupo de enfermedades en el gasto sanitario y nacional por lo que las mismas significan a nivel macroeconómico; se logró develar que los gastos de atención médica para las enfermedades cardiovasculares fue la más alta, los costos de salud relacionados con las ECNT varían según las 
regiones del mundo y de acuerdo con el tipo de enfermedad, los años vividos con ella, puesto que las de mayor gravedad generan los más altos costos; sin embargo, estas podrían ser estimaciones poco precisas pues se reportó que hay escasez de información relativa a la verdadera carga económica de las ECNT principalmente en aquella que se deriva de países de bajos y medianos ingresos(35) como Colombia.

Según la OPS, la carga económica de las ECNT para la región de las Américas representa un costo elevado para la sociedad lo que contribuye a las desigualdades sociales; las estimaciones disponibles de los costos indican una gran carga económica cada vez mayor para las personas, las familias y los sectores público y privado; las complicaciones de las enfermedades no transmisibles causan costos considerables, como aquellos resultantes de la nefropatía diabética y los de cáncer, que tienden a incrementar(40). Para el período 2006-2015, se calculó que la pérdida acumulativa del producto interno bruto (PIB) causada por las cardiopatías, los accidentes cerebrovasculares y la diabetes fue de 13.540 millones de dólares en cuatro países de la Región: Argentina, Brasil, Colombia y México; en Brasil, cuya economía ocupa el segundo lugar en la Región después de los EU, se calculó en 72.000 millones de dólares el costo del tratamiento y la pérdida de productividad causados por cinco enfermedades no transmisibles(41).

Se ha reportado que la cronicidad, la gravedad de los estadios de las enfermedades y la presencia de comorbilidades, incrementan los costos. Así, los pacientes con enfermedad cerebrovascular tienen casi un $40 \%$ más de incremento en los costos en comparación con los casos leves; mientras que los pacientes con cáncer que tienen entre una y tres comorbilidades en la misma etapa de diagnóstico, incrementan los costos. En pacientes con DM, los costos se triplican entre el primero y el séptimo año después del diagnóstico; en ellos, se ha informado un incremento de aproximadamente $52 \%$ en la segunda etapa comparada con la inicial(42). El CDC reportó un costo total anual derivado de las ECNT para los EU de 316.1 billones de dólares entre los años 2012 y 2013; de esto, 126,4 billones de dólares, fueron costos por pérdida de la productividad derivados de muerte prematura $^{(13)}$.

Los altos costos de las ECNT, incluyendo el tratamiento generalmente largo en el tiempo, lleva al deterioro de las dinámicas familiares, a los cambio de roles y ocupaciones para cuidar al enfermo, al daño de la salud del nuevo cuidador y al gasto excesivo de atención, medicamentos, citas con especialistas, nuevas dietas, procedimientos quirúrgicos entre otros que, bajo el sistema en el que operan países como Colombia, deterioran por completo la calidad de vida de estos pacientes y sus familias. En muchos países, los comportamientos poco saludables se producen tanto en los grupos de mayores como los de menores ingresos; sin embargo, los grupos de altos ingresos pueden acceder a los servicios y productos que los protegen de los riesgos más grandes, mientras que los grupos de menores ingresos no pueden permitirse este acceso(31). Esta dinámica afecta consistentemente la calidad de vida de pacientes y familias.

La calidad de vida relacionada con la salud (CVRS) "se hizo popular a partir de los años 80, posiblemente asociado a los cambios de los perfiles epidemiológicos de morbimortalidad que empezaron a notarse dramáticamente en algunos países desarrollados con el incremento cada vez más grande de la esperanza de vida $y$, contemporáneamente, de enfermedades crónicas en porcentajes cada vez mayores de sus habitantes"(30). Durante el año 2010, se condujo en Lima (Perú), un estudio que logró determinar los factores asociados a la CVRS medida a través del SF-36, en pacientes con cardiopatía coronaria y DM. Se encontró que personas mayores de 65 años, mujeres, personas con sobrepeso $u$ obesidad, con hemoglobina glucosilada menor de 7,5 $\mathrm{mg} / \mathrm{dL}, \mathrm{DM}$, antecedente de enfermedad coronaria, comorbilidades y bajo nivel socioeconómico, son factores asociados a la pobre CVRS en pacientes con cardiopatía coronaria y DM siendo aún más baja en el dominio físico para estos últimos(43). 
En general, estudios e informes sobre CVRS en enfermos crónicos se han hecho en enfermedades específicas; gran parte de ellos buscando factores relacionados a la calidad de vida de estos pacientes. A partir de los mismos, se ha documentado que, a nivel psicosocial, la enfermedades crónicas producen altos niveles de estrés teniendo un efecto negativo sobre el ajuste psicológico de las personas que las padecen y requieren un periodo de adaptación de parte del individuo y de la familia(30,44).

En septiembre de 2016, la OMS y la OPS para la región de las Américas, advirtieron que las consecuencias negativas de las ECNT están puestas sobre las condiciones de salud de las personas, sus familias y la comunidad, así como su economía y de los sistemas públicos y privados de las naciones, lo que amenaza el crecimiento económico de América Latina y el Caribe ${ }^{(40)}$. Por tanto, las recomendaciones para su prevención y control van encaminadas hacia la implementación de estrategias altamente costo-efectivas tales como la promoción de la actividad física.

\section{Inactividad física: magnitud del problema}

La actividad física ha sido reconocida como una estrategia de fácil implementación, costoefectiva, no invasiva, equitativa. Ha demostrado innumerables beneficios para la salud general y la calidad de vida en quienes la realizan. Se ha documentado que la realización de actividad física en sus niveles recomendados, es esencial para una buena salud durante toda la vida $(45,46)$. A través de un comunicado de prensa en mayo 6 de 2014, el director del CDC, Dr. Tom Frieden, afirmó que: "La actividad física es lo más parecido que tenemos a una medicina milagrosa"(47) y hace parte de los indicadores de salud y de control de factores de riesgo para las ECNT, como lo muestra la reciente Encuesta Nacional de la Situación Nutricional en Colombia, ENSIN(48).

Una publicación reciente pretendió develar los resultados de las revisiones clave, basadas en la evidencia puestas en un marco socio-ecológico, con el fin de comprender la efectividad de las intervenciones en AF como una de las estrategias de control de ECNT. Esta revisión se llevó a cabo a partir de dos de las guías mundiales más utilizadas por investigadores y profesionales; la serie de Guías de Servicios Comunitarios Preventivos de EU y la de prevención del Instituto Nacional del Reino Unido para la Salud y Atención de Excelencia (NICE), diseñadas con el propósito de guiar los esfuerzos de promoción de la salud y prevención de ECNT, tanto en entornos clínicos como en los comunitarios. La guía comunitaria ha sido culturalmente adaptada para revisar las intervenciones de promoción de AF y prevención de la obesidad en América Latina. Se sintetiza la evidencia de intervenciones en dieta $\mathrm{y}$ en $\mathrm{AF}$ para el tratamiento y la prevención de ECNT dadas en recomendaciones fuertes a favor. Estos enfoques de intervención, incluyen campañas sociales de comportamiento, de información, de atención de salud, así como enfoques ambientales y políticos ${ }^{(49) \text {. }}$

Para que la AF se traduzca en beneficios para la salud y se asocie con la disminución en el riesgo de muerte prematura, de las ECNT y de algunas condiciones musculo-esqueléticas, se sugiere realizarla cumpliendo con los requisitos en intensidad, duración y frecuencia. La recomendación actual, de acuerdo con las guías de actividad física para los americanos del año 2008(50) y de la Organización Mundial de la Salud(16), determina que los adultos de 18 a 64 años deben realizar al menos 150 minutos semanales de actividad física aeróbica de intensidad moderada, o 75 minutos semanales de AF aeróbica vigorosa, o una combinación equivalente a AF aeróbica de intensidad moderada o vigorosa. Esta actividad debe ser realizada en periodos de al menos 10 minutos continuos diarios preferiblemente lo largo de la semana(51,52).

La OMS, declara a la inactividad física como el cuarto factor de riesgo en lo que respecta a la mortalidad mundial $(6 \%$ de las muertes registradas)(1,36). Según la OMS, al menos un $60 \%$ de la población mundial no realiza la $\mathrm{AF}$ necesaria para obtener beneficios en salud, la inactividad física es reconocida como factor predictor de casi todas las enfermedades conocidas como de alto costo y causante de 
muerte prematura(53). En las Américas, el $43 \%$ de la población es inactiva; encuestas nacionales recientes en Brasil reportaron una alta prevalencia de inactividad física (66\% en adultos y $74 \%$ en adultos mayores) ${ }^{(54)}$.

En Colombia, según la Encuesta Nacional de la Situación Nutricional (ENSIN) en 2015, la prevalencia nacional de cumplimiento de actividad física por semana fue tan solo del $51,1 \%{ }^{(48)}$. En entrevista a un diario del país, investigadores expertos en el tema afirman que Colombia es el sexto país latinoamericano al que más se le atribuyen muertes por eventos clínicos originados por la exposición a la inactividad física: "por culpa de la inactividad física mueren al año 5,3 millones de personas en el mundo"(18). Otro estudio realizado en 2011 por Patiño et al.(55), reveló que en Colombia la inactividad física presentó una prevalencia del $56,6 \%$, era el factor de riesgo cardiovascular en la población adulta más frecuente y el cuarto en el porcentaje de riesgo atribuible a la población (RAP) $(15,7 \%)$. Según estudio de 2014 más de la mitad de las personas no realizan actividad física; solo el $42,6 \%$ de los adultos entre los 18 y 64 años hacen la $\mathrm{AF}$ recomendada(56).

Estudios empíricos han mostrado con suficiencia la asociación de los niveles de actividad física con características de los individuos centradas en los factores demográficos (sexo, estrato socioeconómico, nivel educativo)(17,51,57,58), comportamentales (hábitos inadecuados de estilos de vida)(59,60), y aptitudinales (características antropométricas)(61); desde este enfoque se han realizado cuestionamientos que indagan por qué unas personas son más activas que otras, bajo un contexto general que pretende pensar que ésta, es una estrategia de muy bajo costo, de alta eficacia, con cobertura poblacional y con un impacto benéfico, que va más allá de la reducción de los indicadores de morbimortalidad por las ECNT.

La baja prevalencia de la realización de $\mathrm{AF}$, ha buscado analizar este indicador a través de factores individuales. Hay consenso mundial en decir que las personas de sexo femenino, adultos $\mathrm{y}$ adultos mayores, de bajos estratos, de escasos ingresos, y nivel educativo nulo y bajo, además de una mala percepción de su salud general, son quienes tienen mayor probabilidad de ser inactivos $y$ de tener una baja autoeficacia (confianza en la capacidad de ser físicamente activo en situaciones específicas) ${ }^{(27,62) \text {. }}$

Una revisión sistemática del año 2012, se propuso indagar por los factores asociados y determinantes de la $\mathrm{AF}$, pero centrado principalmente en los factores a nivel individual en los países de altos ingresos. Se demostró que, la edad, el sexo, el estado de salud, la autoeficacia y la motivación estuvieron asociados con la realización de actividad física. Además, identificó los determinantes y los factores asociados por grupos de edad, dentro de los que se encontró que ser de sexo masculino fue un factor determinante positivo solo para el grupo de 4 a 9 años. Desde el componente psicosocial, la autoeficacia se asoció positivamente y es un determinante en niños y adolescentes. Los resultados para los factores de conducta en los niños y adolescentes varían: fumar parece no estar relacionado con la $\mathrm{AF}$, pero sí la $\mathrm{AF}$ previa la cual se cataloga como predictor. Respecto a los factores sociales, el apoyo familiar en los adolescentes, se confirmó como un factor determinante para la $\mathrm{AF}^{(17)}$.

En el grupo de adultos, dentro de los factores asociados al componente demográfico, se encontraron la edad, la educación, el sexo, los ingresos, el estado civil, la etnia, el estado de salud percibida, y el índice de masa corporal (IMC). De otro lado, los psicosociales que se reportaron de manera asociada fueron las actitudes, la intención, la planificación, la autoeficacia, etapa del cambio, el estrés, el esfuerzo percibido, la realización de metas, los resultados psicológicos, el apoyo social de amigos y compañeros, y las normas sociales. Sin embargo, estos mismos estudios afirman que aún hay poco conocimiento con respecto a los factores que determinan la realización de la $\mathrm{AF}$ entendiendo que no depende solo de los factores centrados en el individuo(51,63).

Reportes de la OMS, OPS, y el CDC, así como otros estudios a nivel mundial(58,62,64,65) y 
nacional(51,66,67), concuerdan en términos generales con estos hallazgos. Sin embargo, aunque a partir de los mismos se han generado diversas estrategias de intervención, el incremento en la prevalencia de inactividad física sigue avanzado en la medida en que avanzan los procesos de industrialización y globalización; por tanto, surge la necesidad de indagar por otros factores que puedan contribuir con la explicación multicausal de la inactividad física y que generen una toma de decisiones más racional para el control de las enfermedades crónicas derivadas de este factor de riesgo(22).

\section{Actividad física y entornos}

Se ha descrito que algunas teorías de la epidemiología han venido avanzando en el abordaje de las causas del contexto como posibles explicaciones a fenómenos de salud y enfermedad, considerando que en ellos están las causas de las causas y que por tanto, las intervenciones que desde la salud pública se derivan, podrían considerar factores más explicativos y de mayor impacto para el control de condiciones en salud. Uno de estos modelos es la epidemiología social. Aunque se conocen dos corrientes claramente definidas (Anglosajona y Latinoamericana), "la epidemiología social busca ampliar el alcance de la investigación para incluir variables y conceptos extraídos de una teoría en efecto, articulando marcos sociológicos a la investigación epidemiológica"(68).

La epidemiología social estudia la distribución y los determinantes sociales de los estados de salud y proporciona información útil sobre las políticas en materia de desigualdades en salud(23,24). Busca ampliar el alcance de la investigación para incluir variables y conceptos extraídos de una teoría en efecto, articulando marcos sociológicos a la investigación epidemiológica(69). Pioneros de la epidemiología social describen que "los fenómenos colectivos de salud funcionan de manera más parecida a una "caja china", en donde los sistemas de determinación epidemiológica se encuentran separados y organizados jerárquicamente, de forma tal que un sistema abarca varios subsistemas, compuestos a su vez por subsistemas de menor jerarquía"(70). Esto ha traído en los últimos años un incremento en el análisis de factores de diversos niveles que determinan la realización de actividad física de las poblaciones(71). La incorporación de una mirada más integral a las dimensiones biológica, socioeconómica, cultural y política al enfoque epidemiológico ha mejorado el análisis de estos factores $\mathrm{y}$ los avances en las técnicas relacionadas con las mismas(72).

Dados los enfoques propuestos desde diversos modelos epidemiológicos y considerando las características de los países en términos de su contexto (ambiente y lo político), han surgido hipótesis relacionadas con los efectos del contexto sobre los comportamientos individuales que determinan la realización o no de la AF. El comportamiento individual para la realización de la AF tiene múltiples niveles de influencias, incluyendo el intrapersonal (biológico, psicológico), interpersonal (social, cultural), organizacional, comunitario, físico ambiental y político. Por ejemplo, el urbanismo actual ha creado factores ambientales que desalientan la práctica de actividad física, estos, asociados a la gran densidad de tráfico, a la mala calidad del aire, a la inexistencia de instalaciones recreativas, a la superpoblación(73-75).

Las enfermedades no transmisibles, asociadas a la inactividad física son el mayor problema de salud pública en la mayoría de países del mundo; pero detrás de la inactividad física hay condiciones que le impiden ser autónomo para tomar la decisión de ser o no activo físicamente. Dichas condiciones interactúan con las características individuales $\mathrm{y}$, aunque Colombia no cuenta aún con hallazgos contundentes en este sentido, países de altos ingresos han contribuido con la explicación de los efectos de los entornos sobre tales decisiones(76) que si bien, no pueden extrapolarse precisamente por las condiciones del contexto, si pueden ayudar a dilucidar cómo han sido los procesos en la interacción del contexto y las características individuales en la realización de la AF.

Los modelos ecológicos tienen una visión amplia de la causalidad centrada en comportamientos de salud, y determina al entorno social y físico 
como contribuyentes a la falta de actividad física, sobre todo los que están fuera del sector de la salud, tales como la planificación urbana, los sistemas de transporte, parques y senderos. Nuevas áreas de investigación determinantes han explorado el seguimiento longitudinal de la actividad física durante toda la vida e identificado factores genéticos que contribuyen a la propensión a ser físicamente activo, factores evolutivos y la obesidad que pueden predisponer a la inactividad. La comprensión de los correlatos y determinantes, especialmente en países de ingresos bajos y medios, podría reducir el efecto de futuras epidemias de inactividad y contribuir a la prevención eficaz a nivel mundial de las enfermedades no transmisibles(20).

Bajo el contexto de los países de altos ingresos de donde proviene la mayor proporción de estudios en este tema, investigadores han propuesto el enfoque de modelos multinivel para la promoción de la actividad física. Derivado del concepto y de la evidencia que demuestra sus condicionantes, estos autores proponen que los mecanismos para la realización de la $\mathrm{AF}$, refleja "el juego" entre ésta, el medio ambiente y la política, y que éste juego responde a un marco teórico de tipo multidimensional. El modelo muestra cómo el nivel de comportamiento de salud individual, entendida como una habilidad personal para realizar AF, está entrelazada no sólo con las estructuras tales como el medio ambiente, sino también con niveles de "elección colectiva" de la formulación de las políticas; es decir, la decisión autoritaria sobre regulaciones que afectan a las decisiones de la población, por ejemplo, el acceso de ciertos grupos a instalaciones deportivas(77). Lo anterior, motivado también por los debates en función de la desigualdad, que en torno a la salud pública se han generado.

La epidemiologia sociocultural se caracteriza por la integración de procesos sociales, culturales, económicos y políticos con procesos biológicos en el estudio de los determinantes de la salud(24). Por tanto, evaluar de manera conjunta estos factores sobre la determinación de conductas saludables o no, cobra relevancia en cuanto permite determinar cómo operan las interacciones entre estos factores para comprender algunos fenómenos en salud; algunos tan complejos como la actividad física, que podrían ayudar a develar explicaciones de las conductas saludables y el éxito o fracaso de las intervenciones para mejorar los indicadores.

Bajo este enfoque, en las últimas décadas se ha venido incorporando este concepto en el estudio de los niveles de actividad fisca de la población, buscando encontrar explicaciones de la baja prevalencia de la misma, hecho que se ha asociado con la persistencia de las ECNT. Paradójicamente, aunque las ECNT son un problema de salud pública en prácticamente todas las regiones del mundo, no es tan grave en los países de altos ingresos si se compara con los de medianos y bajos ingresos pero es de los primeros, de donde proviene la mayor parte de la literatura que puede mostrar con suficiencia los efectos del contexto sobre la realización de actividad física recomendada(78-80).

En Colombia un grupo de investigadores ha venido trabajando el tema en conjunto con redes internacionales dedicadas al estudio de la $\mathrm{AF}$ y por tanto, los acercamientos a este nuevo abordaje podrían ser un modelo para el estudio de contexto en países con bajos y medianos ingresos. Un estudio multinivel realizado en Bogotá-Colombia, sugirió que las características de la zona residencial se asociaban con la percepción del uso de parques para realizar AF, aunque también se encontró que el medio ambiente circundante, tiene una relación inversa entre la conectividad y la $\mathrm{AF}$, lo que pone de manifiesto la necesidad de intervenciones en el ámbito del tráfico y para la seguridad de los peatones. Se encontró que, en comparación con las personas inactivas, los que residen en barrios ubicados en el tercil superior según la densidad de los parques tenían dos veces más probabilidad de realizar $\mathrm{AF}$ regular $(\mathrm{OR}=2,05$; IC 95\%: 1,13 a 3,72; p=0,021). Así también, evaluando la disponibilidad de medios de transporte, se encontró que aquellos que residían cerca de estaciones de Transmilenio (sistema de transporte masivo), eran menos propensos a la realización de la $\mathrm{AF}(\mathrm{OR}=1,27$; IC 95\%: 1,07 a 1,50; $\mathrm{p}=0,009$ ). El estudio concluye 
que, para hacer frente a la inactividad física se deben considerar las influencias potenciales de planificación urbana y sistemas de transporte masivo $^{(45)}$.

Algunos países vienen explorando desde inicios de la década del 2000, relaciones entre características de los entornos con la realización de actividad física. En el año 2002, se publicó un estudio sobre la relación entre la actividad física y la autopercepción de la salud en adultos mayores, tanto a nivel de barrio como a nivel de los residentes, la hipótesis del estudio fue que existe un conjunto de predictores relacionados con los niveles de actividad física a nivel de barrio, siendo estos: la cohesión social, la proximidad de la vivienda a instalaciones para realizar actividad física, la seguridad para caminar en el barrio y la importancia de la participación en actividad física; los autores consideraron como factores de confusión a nivel de residente, aquellas relacionadas con el género, la edad, el nivel de educación, los ingresos del hogar, y el grado de confianza en sí mismo para caminar. El estudio encontró que el 15\% del estado de salud y el $28 \%$ de la actividad física fue debido a las condiciones del entorno. Los resultados indicaron que los altos niveles de cohesión social $(\beta=0,33)$, la proximidad a instalaciones para realizar actividad física en el barrio $(\beta=0,28)$, una buena percepción de seguridad para caminar en el barrio $(\beta=0,20), y$ la percepción de la importancia de la participación en actividad física a nivel de barrio $(\beta=0,37)$, estuvieron relacionadas con altos niveles de actividad física(81).

Otro estudio similar encontró que las características del entorno físico, se asociaron con la caminata en adultos mayores, el $28 \%$ de dichos niveles de caminata fueron atribuidos a las diferencias entre los barrios. Así, hay evidencia para concluir que existen diferencias entre los barrios respecto a la realización de actividad física. También, se muestra que los barrios con alta densidad de lugares laborales y de viviendas, así como un mayor número de espacios verdes y abiertos para la recreación, están relacionados positivamente con la caminata. Un mayor número de intersecciones de las calles contribuyó al incremento de la caminata, solo cuando los residentes percibían que las condiciones del tráfico en dichas intersecciones eran seguras; en esta relación, medió la percepción de seguridad de los habitantes entre el entorno físico y los niveles de caminata(82).

Otro estudio de Estados Unidos en 2006, mostró un modelo predictivo en 267 adultos mayores con hipertensión arterial (HTA) limítrofe; el modelo teórico propuesto incluyó variables que obedecieron a los constructos de características individuales, interpersonales y del entorno, para probar su influencia en los niveles de actividad física y que una vez obtenidos los resultados se explicó que el $44 \%$ de la realización de actividad física fue variabilidad atribuida a condiciones del entorno. Las variables que predijeron directamente la $\mathrm{AF}$ fueron el género, los ingresos, la experiencia previa en el ejercicio, la autoeficacia y motivación para la AF; las variables que predijeron indirectamente la $\mathrm{AF}$ fueron la percepción del estado de salud, las barreras para la realización de AF, la autoeficacia, la motivación intrínseca para la salud, y la influencia del medio ambiente(83).

Un estudio reciente en Canadá (2016) probó la hipótesis de que los ambientes del barrio o del vecindario, pueden apoyar $\mathrm{u}$ obstaculizar la actividad física; los autores proponen teorías psicológicas del cambio de comportamiento de la salud en el contexto de la investigación del entorno físico para comprender mejor la interacción de las características ambientales y psicosociales que determinan comportamientos sedentarios y la realización de actividad física. Se encontró relación negativa entre la percepción de la conectividad de la calle y el uso del suelo mixto con el comportamiento sedentario; del mismo modo, hubo relación entre los efectos de la conectividad de la calle y el uso de suelo sobre la AF. Los resultados mostraron la importancia que tiene la percepción del entorno físico con la $\mathrm{AF}$ y el comportamiento sedentario, en gran parte debido a que estas percepciones ambientales están vinculadas de manera positiva a la confianza para caminar( ${ }^{84)}$. 
En Alemania (2011), un estudio de caso de un proyecto de promoción de la salud a nivel local se utilizó para ilustrar el poder explicativo de un modelo multidimensional, mostrando cómo la interacción entre las estructuras, las agencias de funcionamiento político y el nivel de elección colectiva condujo a la creación de una piscina cubierta municipal solo para mujeres, generando un impacto importante en el aumento de los niveles de actividad física y en la mejora de la autoeficacia general entre los miembros del grupo objetivo(77).

Para enunciar un ejemplo del contexto latinoamericano, un estudio cross-sectional en Brasil (2009), se propuso indagar en 385 adultos mayores, la asociación entre la realización de AF en el tiempo libre y la percepción sobre el entorno físico; la proporción de personas activas durante el tiempo libre, fue tan solo del 15,2\%. En los hombres, una mayor realización de AF se asoció con: la presencia de pistas deportivas $(\mathrm{OR}=2,95)$, disponibilidad de bancos para ir caminando $(\mathrm{OR}=3,82)$ y las clínicas de salud $(\mathrm{OR}=3,60)$; también se encontró asociación con la buena percepción de seguridad durante el día $(\mathrm{OR}=4,21)$ y la invitación de los amigos para hacer ejercicio $(\mathrm{OR}=3,13)$. En las mujeres la realización de $\mathrm{AF}$ se asoció con la presencia de las iglesias o templos religiosos $(\mathrm{OR}=5,73)$, disponibilidad de gimnasios $(\mathrm{OR}=2,49)$ y de plazas $(\mathrm{OR}=3,63)$. Esto pone de manifiesto que, existen diferencias en percepciones según el sexo y que para ofertar programas para promover la actividad física para la tercera edad, se deben considerar las variables asociadas con estructuras públicas y privadas tales como disponibilidad de gimnasios, plazas, canchas deportivas, clínicas de salud y bancos; así como lugares donde haya reuniones sociales (iglesias, grupos comunitarios), y los que favorezcan el apoyo social tales como la invitación de amigos a hacer ejercicio. La percepción de la seguridad es un factor que explica los niveles de $\mathrm{AF}$ en el tiempo libre de este grupo poblacional(85).

Muchos de estos estudios, han servido como referentes para la toma de decisiones en países de altos ingresos, mejorando los niveles de actividad física de la población. Por ejemplo, en
Las Vegas, Estados Unidos (2014), se condujo un estudio descriptivo que utilizó datos de observación para evaluar la relación de los determinantes ambientales y sociales con los niveles de intensidad de la $A F$ en jóvenes que acuden a parques de los barrios de la ciudad; la alta velocidad del tráfico $(\mathrm{OR}=0,76)$, la mala condición de aceras $(\mathrm{OR}=0,34)$ y los barrios de bajos ingresos $(\mathrm{OR}=0,07)$ se asociaron con probabilidades de disminución de $A F$ vigorosa( ${ }^{86)}$.

En Carolina del Norte, se publicó en 2014, el informe sobre los efectos de la predicción de un diseño urbano para cuantificar los beneficios en salud, a partir de éste se generaron propuestas al diseño que están altamente relacionadas con la AF como: la creación de mayor conciencia sobre el papel de la infraestructura peatonal y de ciclorutas en las decisiones de las personas para hacer AF, gestionar la financiación con el gobierno y organizaciones de desarrollo económico para la construcción de aceras, pues a pesar del alto flujo de peatones, estas no existían; también se sugirió evaluar el grado de compromiso de los residentes para las mejoras en los barrios que incluye realizar auditorías de transitabilidad con los vecinos para catalogar la infraestructura existente, identificar las áreas prioritarias para la base de las actividades de promoción, realizar ejercicios de mapeo mental con los vecinos para entender qué desean o no de las características del barrio, acercamiento a otros grupos que apoyan las caminatas y el uso de las ciclo-rutas y lograr el compromiso para que los departamentos de transporte promocionen las estrategias de $\mathrm{AF}^{(87)}$.

En términos generales, se ha descrito que la disponibilidad de medios de transporte para ir a lugares a hacer $\mathrm{AF}$, la percepción de seguridad, la estética del barrio, un ambiente positivo en los lugares donde se reside, el estado adecuado de aceras, la cercanía desde las casas a lugares de interés para las personas, la densidad de parques, las condiciones para caminar, una menor disponibilidad de transporte público, la infraestructura, el estrato socioeconómico alto, entre otras, están asociados con mayor seguridad e interés para hacer AF en los barrios donde se 
reside $\mathrm{o}$ en aquellos cercanos(78,80,88). También están relacionados factores como la aplicación de políticas integrales de promoción, percepción positiva de participación de gobiernos locales, acción comunitaria, incremento en las políticas, la participación comunitaria(17,75,89).

\section{Conclusiones}

Esta revisión permitió acoger los elementos clave de las enfermedades crónicas dentro de los que se logró incluir los efectos negativos desde la salud hasta la economía de pacientes, familias y el sistema, y describir los principales factores de riesgo priorizando la inactividad física como uno de los factores determinantes en su aparición. A partir de esto, se hizo una exploración de la evidencia sobre los factores asociados a la inactividad física como conducta no saludable y se encuentra que, adicional a ciertas características individuales, el papel que desempeñan los entornos construidos y percibidos en que habitan las personas es un determinante para la realización, o no, de actividad física y este a su vez, para la explicación de la prevención y control de las enfermedades crónicas, lo que explica la necesidad de incorporar diversos niveles que influencian las conductas de las personas para indagar por las causas que conlleven a la implementación de estrategias de intervención más razonables.

Aunque el problema de las enfermedades crónicas ha logrado despertar a nivel mundial en investigadores y clínicos el interés por su estudio y su manejo, las estrategias para incrementar su realización son aún ineficaces. A pesar del conocimiento que se tiene sobre los beneficios de la actividad física, la prevalencia de esta actividad es muy baja en todo el mundo. La evidencia que proviene de países de altos ingresos ha ayudado a develar otros mecanismos que pudieran explicar las condiciones de realización de actividad física en países como Colombia pero, dadas las diferencias contextuales y la dificultad de generalizar los resultados, se hacen necesarios estudios que muestren a través de análisis robustos, los determinantes de esta intervención costo- efectiva para el control de las crecientes enfermedades crónicas.

\section{Referencias}

1. Organización Mundial de la Salud. Action Plan for the Global Strategy for the Prevention and Control of Noncommunicable Diseases. Ginebra: OMS; 2008.

2. Observatorio Nacional de Salud. Informe técnico. Carga de enfermedad por Enfermedades Crónicas No Transmisibles y Discapacidad en Colombia. Bogotá: ONS; 2015. p. 222.

3. Ministerio de Salud y Protección Social. Prevención de Enfermedades no transmisibles. Bogotá: MinSalud; $2007 . \quad$ Disponible en: http://www.minsalud.gov.co/salud/publica/Paginas/s alud-publica.aspx

4. Organización Mundial de la Salud. Prevención de las enfermedades no transmisibles en el lugar de trabajo a través del régimen alimentario y la actividad física. Informe de la OMS y el Foro Económico Mundial sobre un evento conjunto. Ginebra: OMS; 2008. Disponible en: http://www.who.int/dietphysicalactivity/WEFreport_s panish.pdf

5. Pearson TA, Blair SN, Daniels SR, Eckel RH, Fair JM, Fortmann SP, et al. AHA Guidelines for Primary Prevention of Cardiovascular Disease and Stroke: 2002 Update: Consensus Panel Guide to Comprehensive Risk Reduction for Adult Patients Without Coronary or Other Atherosclerotic Vascular Diseases. American Heart Association Science Advisory and Coordinating Committee. Circulation. 2002;106(3):388-91.

6. World Health Organization. Actividad física. Report No: 384. Geneva: WHO; 2014.

7. Bauman A, Phongsavan P, Schoeppe S, Owen N. Medición de actividad física: una guía para la promoción de la salud. Glob Health Promot. 2006;13(2):92-103.

8. Garber CE, Blissmer B, Deschenes MR, Franklin BA, Lamonte MJ, Lee IM, et al. Quantity and quality of exercise for developing and maintaining cardiorespiratory, musculoskeletal, and neuromotor fitness in apparently healthy adults: guidance for prescribing exercise. Med Sci Sports Exerc. 2011;43(7):1334-59.

9. Vidarte JA, Vélez C, Sandoval C, Alfonso M. Actividad física: estrategia de promoción de la salud. Hacia Promoc Salud. 2011;16(1):202-218.

10. Organización Panamericana de la Salud. Prevención y control de enfermedades. Enfermedades no transmisibles. Ginebra: OPS; 2008.

11. Bauer UE, Briss PA, Goodman RA, Bowman BA. Prevention of chronic disease in the 21st century: elimination of the leading preventable causes of premature death and disability in the USA. Lancet Lond Engl. 2014;384(9937):45-52.

12. World Health Organization. Assessing national capacity for the prevention and control of noncommunicable diseases: report of the 2010 global survey. Geneva: WHO; 2012. 
13. Centers for Disease Control and Prevention. Chronic Disease Prevention and Health Promotion: The Cost of Chronic Diseases and Health Risk Behaviors. Estados Unidos: CDC; 2017. [citado 19 de febrero de 2018]. Disponible en: https://www.cdc.gov/chronicdisease/overview/

14. Organization mondiale de la santé. Global status report on noncommunicable diseases 2014: attaining the nine global noncommunicable diseases targets; a shared responsibility. Geneva: World Health Organization; 2014. Disponible en: http://www.who.int/nmh/publications/ncd-statusreport-2014/en/

15. Institute for Health Metrics and Evaluation, Human Development Network, The World Bank (Instituto para la Medición y Evaluación de la Salud). La carga mundial de morbilidad: generar evidencia, orientar políticasEdición regional para América Latina y el Caribe. Seattle: IHME; 2013.

16. Organización Mundial de la Salud. Estrategia mundial sobre régimen alimentario, actividad física y salud. Inactividad física: un problema de salud pública mundial. Ginebra: OMS; 2015. Disponible en: http://www.who.int/dietphysicalactivity/factsheet_ina ctivity/es/

17. Bauman AE, Reis RS, Sallis JF, Loos RJ, Martin BW, et al. Correlates of physical activity: why are some people physically active and others not?. The lancet. 2012;380(9838):258-271.

18. Redacción Vivir. Colombia, país sedentario. Bogotá: El Espectador; 2012. Disponible en: http://www.elespectador.com/noticias/actualidad/viv ir/colombia-pais-sedentario-articulo-367555

19. Laine J, Kuvaja-Köllner V, Pietilä E, Koivuneva M, Valtonen H, Kankaanpää E. Cost-Effectiveness of Population-Level Physical Activity Interventions: A Systematic Review. Am J Health Promot. 2014;29(2):71-80.

20. Kohl HW, Craig CL, Lambert EV, Inoue S, Alkandari JR, Leetongin $\mathrm{G}$, et al. The pandemic of physical inactivity: global action for public health. The Lancet. 2012;380(9838):294-305.

21. Crespo C, Escutía G, Salvo D, Arredondo E. Determinantes de la disminución de la actividad física y el aumento del sedentarismo. In: Rivera Dommarco J, Hernández Avila M, Aguilar-Salinas C, Vadillo-Ortega F, Murayama-Rendón C. Obesidad en México Recomendaciones para una política de Estado. Ciudad de México: Universidad Nacional Autónoma de México; 2012.

22. Vélez EFA, Villada FAP, Cardona GD. Factores asociados con la adherencia a la actividad física en el tiempo libre. Educ Física Deporte. 2014;33(1):129-151.

23. Diez AV. A glossary for multilevel analysis. J Epidemiol Community Health. 2002;56(8):588.

24. Diex AV. La necesidad de un enfoque multinivel en epidemiología. Región Soc. 2008;XX(2):77-91.

25. Roux AD. Complex systems thinking and current impasses in health disparities research. Am J Public Health. 2011;101(9):1627-1634.
26. Ministerio de Salud. ¿Qué se sabe sobre la inactividad física y las enfermedades no transmisibles?. Bogotá: MinSalud; 2018. Disponible en: http://www.minsalud.gov.co/proteccionsocial/Paginas /Actividad-F\%C3\%ADsica.aspx

27. Bonomi AG, Plasqui G, Goris AHC, Westerterp KR. Aspects of activity behavior as a determinant of the physical activity level: Behavior and activity level. Scand J Med Sci Sports. 2012;22(1):139-45.

28. World Health Organization. Global recommendations on physical activity for health. Geneva: WHO; 2010.

29. The Center for managing Chronic disease. What is Chronic Disease?. Ann Arbor: University of Michigan; 2011. Disponible en: http://cmcd.sph.umich.edu/whatis-chronic-disease.html

30. Vinaccia S, Quiceno JM. Calidad de Vida Relacionada Con La Salud y Enfermedad Crónica: Estudios Colombianos. Psychol Av Discip. 2012;6(1):123-36.

31. World Health Organization. Noncommunicable diseases. Geneva: OMS; 2017. Disponible en: http://www.who.int/mediacentre/factsheets/fs355/e $\mathrm{n} /$

32. Organización Mundial de la Salud. Enfermedades crónicas. Ginebra: OMS; 2018. Disponible en: http://www.who.int/topics/chronic_diseases/es/

33. Centers for Disease Control and Prevention. Chronic Diseases: The leading causes of death and disability in the United States. United States: CDC; 2015. Disponible en: http://www.cdc.gov/chronicdisease/overview/

34. Centers for disease control and prevention. Chronic Disease Prevention System. United States: CDC; 2014. Disponible http://www.cdc.gov/chronicdisease/about/prevention .htm

35. Muka T, Imo D, Jaspers L, Colpani V, Chaker L, van der Lee SJ, et al. The global impact of non-communicable diseases on healthcare spending and national income: a systematic review. Eur J Epidemiol. 2015;30(4):251-77.

36. Sallis JF, Cerin E, Conway TL, Adams MA, Frank LD, Pratt $M$, et al. Physical activity in relation to urban environments in 14 cities worldwide: a cross-sectional study. The Lancet. 2016;387(10034):2207-2217.

37. Krieger N. Theories for social epidemiology in the 21 st century: an ecosocial perspective. Int J Epidemiol. 2001;30(4):668-77.

38. Organización Mundial de la Salud. Enfermedades no transmisibles. Ginebra: OMS; 2017. [Citado 10 de marzo de 2018]. Disponible en: http://www.who.int/mediacentre/factsheets/fs355/es /

39. Organización Mundial de la Salud. Enfermedades crónicas y promoción de la salud: prevención de las enfermedades crónicas. Ginebra: OMS; 2018. [Citado 10 de marzo de 2018]. Disponible en: http://www.who.int/chp/chronic_disease_report/part 1/es/index7.html

40. Legetic B, Medici A, Hernández-Ávila M, Alleyne G, Hennis A. Las dimensiones económicas de las enfermedades no transmisibles en América Latina y el Caribe. Washington: OPS; 2017. 
41. Organización Panamericana de la Salud. La carga económica de las enfermedades no transmisibles en la región de las Américas. Informe temático sobre enfermedades no transmisibles. Ginebra: OPS; 2011. Disponible en: http://www.paho.org/hq/index.php?option=com_doc man\&task=doc_view\&gid=14371\&Itemid=270\&lang=e $\mathrm{n}$

42. Kohl HW, Craig CL, Lambert EV, Inoue S, Alkandari JR, Leetongin $\mathrm{G}$, et al. The pandemic of physical inactivity: global action for public health. Lancet Lond Engl. 2012;380(9838):294-305.

43. Barrantes M. Factores asociados a la calidad de vida relacionada a la salud en pacientes con cardiopatía coronaria y diabetes mellitus. Rev Med Hered. 2010;21(3):118-27.

44. Dobbie M, Mellor D. Chronic illness and its impact: considerations for psychologists. Psychol Health Med. octubre de 2008;13(5):583-90.

45. Parra DC, Gomez LF, Fleischer NL, David Pinzon J. Built environment characteristics and perceived active park use among older adults: results from a multilevel study in Bogotá. Health Place. 2010;16(6):1174-81.

46. Massidda M, Cugusi L, Mathieu A. Physical activity levels and health-related quality of life in young Italian population. J Sports Med Phys Fitness. 2015;55(5):50612.

47. Frieden C. Inactivity Related to Chronic Disease in Adults with Disabilities Half of adults with disability get no aerobic physical activity. [Internet]. United States: Centers for disease control and prevention; 2014. Disponible

en: http://www.cdc.gov/spanish/mediosdecomunicacion/ comunicados/p_adultos_discapacidades_050614.html

48. Ministerio de Salud, Instituto Colombiano de Bienestar Familiar, Instituto Nacional de Salud, Universidad Nacional. Encuesta Nacional de la Situación Nutricional ENSIN 2015. Bogotá: MinSalud; 2018. Disponible en: https://www.minsalud.gov.co/sites/rid/Lists/Bibliotec aDigital/RIDE/VS/ED/GCFI/presentacion-ensin-2017medellin.pdf

49. Pratt M, Perez LG, Goenka S, Brownson RC, Bauman A, Sarmiento OL, et al. Can population levels of physical activity be increased? Global evidence and experience. Prog Cardiovasc Dis. 2015;57(4):356-67.

50. U.S. Department of Health and Human Services (HHS) Physical Activity Guidelines for Americans. United States: HHS; $2008 . \quad$ Disponible en: www.health.gov/paguidelines/guidelines

51. González S, Sarmiento OL, Lozano O, Ramírez A, Grijalaba C. Niveles de actividad física de la población colombiana: desigualdades por sexo y condición socioeconómica. Biomédica [Internet]. [citado $4 \mathrm{de}$ marzo de 2015];34(3):447-59. Disponible en: http://www.revistabiomedica.org/index.php/biomedic a/article/view/2258

52. Bauman A, Phongsavan P, Schoeppe S, Owen N. Physical activity measurement-a primer for health promotion. Promot Educ. 2006;13(2):92-103.

53. Lee IM, Shiroma EJ, Lobelo F, Blair SN, Katzmarzyk PT. Effect of physical inactivity on major non- communicable diseases worldwide: an analysis of burden of disease and life expectancy. The Lancet. 2012;380(9838):219-29.

54. Parra DC, Hoehner CM, Hallal PC, Reis RS, Simoes EJ, Malta DC, et al. Scaling up of physical activity interventions in Brazil: how partnerships and research evidence contributed to policy action. Glob Health Promot. 2013;20(4):5-12.

55. Patiño FA, Arango E, Quintero $M$, Arenas $M$. Cardiovascular risk factors in an urban Colombia population. Rev Salud Pública. 2011;13(3):433-45.

56. González SA, Sarmiento OL, Cohen DD, Camargo DM, Correa JE, Páez DC, et al. Results from Colombia's 2014 Report Card on Physical Activity for Children and Youth. J Phys Act Health. 2014;11(suppl 1):S33-44.

57. Kaupuzs A. A cross-sectional analysis of physical activity, psychological determinants and health related variables of latvian older adults. Eur J Adapt Phys Act [Internet]. 2012 [citado 4 de marzo de 2015];5(1):39$52 . \quad$ Disponible en: http://eujapa.upol.cz/index.php/EUJAPA/article/view $/ 75$

58. Amireault S, Godin G, Vézina-Im L-A. Determinants of physical activity maintenance: a systematic review and meta-analyses. Health Psychol Rev. 2013;7(1):55-91.

59. Lema L, Salazar I, Varela M, Tamayo J, Rubio A, Botero A. Comportamiento y salud de los jóvenes universitarios: satisfacción con el estilo de vida. Pensam Psicológico. 2009;5(12):71-88.

60. Centers for Disease Control and Prevention. CountyLevel Estimates for Leisure-Time Physical Inactivity [Internet]. United States: CDC; 2011. [citado 23 de enero de 2014]. Disponible en: http://www.cdc.gov/diabetes/pubs/inactivity.htm

61. Prieto D, Correa J, Ramírez R. Niveles de actividad física, condición física y tiempo en pantallas en escolares de Bogotá, Colombia: Estudio FUPRECOL. Nutr Hosp. 2015;32(5):2184-2192.

62. Lavielle P, Pineda V, Jáuregui O, Castillo M. Actividad física y sedentarismo: Determinantes sociodemográficos, familiares y su impacto en la salud del adolescente. Rev Salud Pública. 2014;16(2):161-72.

63. Martínez E, Saldarriaga JF, Sepúlveda FÉ. Physical Activity in Medellín: a Challenge for Health Promotion. Rev Fac Nac Salud Pública. 2008;26(2):117-123.

64. World Health Organization. Assessing national capacity for the prevention and control of noncommunicable diseases: report of the 2010 global survey. Geneva: WHO; 2012.

65. Cansino K, Gálvez H. Determinantes de la participación en actividades físicas en el Perú. Rev Peru Med Exp Salud Pública. 2014;31(1):151-155.

66. González SA, Sarmiento OL, Cohen DD, Camargo DM, Correa JE, et al. Results from Colombia's 2014 Report Card on Physical Activity for Children and Youth. J Phys Act Health. 2014;11(Suppl 1):S33-44.

67. Ministerio de Salud. Plan Decenal de Salud Pública 2012-2021. Dimensión vida saludable y condiciones no transmisibles. Bogotá: MinSalud; 2013.

68. Segura del Pozo J. Epidemiología de campo y epidemiología social. Gac Sanit. 2006;20(2):153-158. 
69. Kubzansky LD, Subramanian SV, Kawachi I, Fay ME, Soobader M-J, Berkman LF. Neighborhood contextual influences on depressive symptoms in the elderly. Am J Epidemiol. 2005;162(3):253-60.

70. Hernández-Ávila M. Epidemiología: diseño y análisis de estudios. Buenos Aires: Editorial Médica Panamericana; 2011.

71. Susser M, Susser E. Choosing a Future for Epidemiology. 1996. Am J Public Health. 2015;105(7):1313-5.

72. Glanz K, Rimer BK, Viswanath K, editores. Health behavior and health education: theory, research, and practice. 4th ed. San Francisco: Jossey-Bass; 2008. 552 p.

73. Salvo D, Reis RS, Stein AD, Rivera J, Martorell R, Pratt M. Characteristics of the Built Environment in Relation to Objectively Measured Physical Activity Among Mexican Adults, 2011. Prev Chronic Dis [Internet]. 2014 [citado 4 de abril de 2015];11:140047. Disponible en: http://www.cdc.gov/pcd/issues/2014/14_0047.htm

74. Ruetten A, Frahsa A, Engbers L, Gusi N, Mota J, et al. Supportive Environments for Physical Activity, Community Action, and Policy in 8 European Union Member States: Comparative Analysis and Specificities of Context. J Phys Act Health. 2014;11(5):873-83.

75. Fernhall B, Borghi-Silva A, Babu AS. The Future of Physical Activity Research: Funding, Opportunities and Challenges. Prog Cardiovasc Dis. 2015;57(4):299-305.

76. Sallis JF, Cain KL, Conway TL, Gavand KA, Millstein RA, Geremia CM, et al. Is Your Neighborhood Designed to Support Physical Activity? A Brief Streetscape Audit Tool. Prev Chronic Dis. 2015;12:150098.

77. Rütten A, Gelius P. The interplay of structure and agency in health promotion: Integrating a concept of structural change and the policy dimension into a multi-level model and applying it to health promotion principles and practice. Soc Sci Med. 2011;73(7):953-9.

78. Liu Q, Ren Y, Cao C, Su M, Lyu J, Li L. Association between walking time and perception of built environment among urban adults in Hangzhou. Zhonghua Liu Xing Bing Xue Za Zhi Zhonghua Liuxingbingxue Zazhi. 2015;36(10):1085-8.

79. Hino AAF, Reis RS, Sarmiento OL, Parra DC, Brownson RC. The built environment and recreational physical activity among adults in Curitiba, Brazil. Prev Med. 2011;52(6):419-22.

80. Brázdová ZD, Klimusová H, Hruška D, Prokopová A, Burjanek A, Wulff KRS. Assessment of Environmental Determinants of Physical Activity: a Study of Built Environment Indicators in Brno, Czech Republic. Cent Eur J Public Health. 2015;23 Suppl:S23-9.

81. Li F, Fishe KJ. A multilevel path analysis of the relationship between physical activity and self-rated health in older adults. J Phys Act Health. 2004;1:398412.

82. Li F. Multilevel modelling of built environment characteristics related to neighbourhood walking activity in older adults. J Epidemiol Community Health. 2005;59(7):558-64.

83. Lee Y-S, Laffrey SC. Predictors of physical activity in older adults with borderline hypertension. Nurs Res. 2006;55(2):110-120.
84. Fleig L, Ashe MC, Voss C, Therrien S, Sims-Gould J, McKay HA, et al. Environmental and psychosocial correlates of objectively measured physical activity among older adults. Health Psychol. 2016;35(12):13641372.

85. Salvador EP, Florindo AA, Reis RS, Costa EF. Perception of the environment and leisure-time physical activity in the elderly. Rev Saúde Pública. 2009;43(6):972-80.

86. Coughenour C, Coker L, Bungum TJ. Environmental and Social Determinants of Youth Physical Activity Intensity Levels at Neighborhood Parks in Las Vegas, NV. J Community Health. 2014;39(6):1092-6.

87. MacDonald J, Rodriguez D, Dennerlein T, Mead J, Bevington S. Predicting Effects of Urban Design on Public Health: A case study in Raleigh, North Carolina. North Carolina: University of North Carolina. Chapel Hill: University of North Carolina; 2014. p. 71.

88. Salvo D, Reis RS, Stein AD, Rivera J, Martorell R, Pratt M. Characteristics of the Built Environment in Relation to Objectively Measured Physical Activity Among Mexican Adults, 2011. Prev Chronic Dis [Internet]. 2014 [citado 15 de febrero de 2016];11:140047. Disponible en: http://www.cdc.gov/pcd/issues/2014/14_0047.htm

89. Gomes, TN, Dos Santos, FK, Zhu, W., Eisenmann, J. y Maia, JAR. Multilevel Analyses of School and Children's Characteristics Associated With Physical Activity. J Sch. octubre de 2014;84(10):668-76. 